\title{
Relapse of idiopathic pulmonary haemorrhage and glomerulonephritis associated with cytomegalovirus (CMV) infection
}

\author{
C.N. Chan, F.M. Lai, K.N. Lai and J.A. Pang \\ Department of Medicine and Department of Morbid Anatomy, The Chinese University of Hong Kong, Prince \\ of Wales Hospital, Shatin, NT, Hong Kong.
}

\begin{abstract}
Summary: A 49 year old man who developed idiopathic pulmonary haemorrhage and glomerulonephritis responded initially to immunosuppressive treatment and plasmapheresis. However, he died later from massive pulmonary haemorrhage and necropsy showed cytomegalovirus infection of the lungs. We propose that relapse of pulmonary haemorrhage may be associated with cytomegalovirus infection and that preventive measures against acquiring the virus through blood products should be considered for elective transfusions in view of the relatively good long term prognosis of this syndrome.
\end{abstract}

\section{Introduction}

In 1919, Goodpasture first described the clinical syndrome of intrapulmonary haemorrhage and glomerulonephritis. ${ }^{1}$ Subsequently, Lerner et al. identified the anti-glomerular basement membrane (anti-GBM) antibody as an important aetiological factor in the pathogenesis of the syndrome. ${ }^{2}$ Thomas \& Irwin first classified diffuse pulmonary haemorrhage on an immunopathological basis depending on the presence or absence of anti-GBM antibody or immune complexes. ${ }^{3}$ A recent review of 40 patients with pulmonary haemorrhage and glomerulonephritis (PHG) identified 3 subgroups: anti-GBM disease, systemic vasculitis and idiopathic. ${ }^{4}$ Association of PHG with influenza $\mathrm{A}_{2}$ virus was reported by Wilson \& Smith, ${ }^{5}$ who proposed that viral infection may induce antibodies reactive with basement membrane which further perpetuate the disease. Rees et al. ${ }^{6}$ later reported the association between bacterial infection and relapse in anti-GBM disease and suggested that intercurrent infection enhanced antibody-mediated tissue injury either through cellular and humoral

Correspondence: J.A. Pang, M.A., M.D., M.R.C.P., Department of Medicine, The Chinese University of Hong Kong, Prince of Wales Hospital, Shatin, NT, Hong Kong. Accepted: 2 March 1987 responses or superimposition of infection-derive immune complex disease. A similar phenomenon was also observed in cases of relapse in Wegener's granulomatosis. ${ }^{7}$ We report a case of a 49 year old man with the idiopathic variety of the syndrome who made a good initial recovery with aggressive treatment but subsequently suffered a fatal relapse associated with cytomegalovirus (CMV) infection. This raises the possibility that, like influenza $A_{2}$ and bacterial infection, CMV may have a role in precipitating a relapse of pulmonary haemorrhage. Since CMV infection may be acquired through blood transfusion, ${ }^{8}$ a treatment frequently used in such patients, there may be grounds for routine screening of blood products for viral contamination before administration.

\section{Case report}

A 49 year old man with a history of rheumatic mitral stenosis was found to have duodenitis on endoscopy in October 1985. His haemoglobin and renal function were both normal.

He presented in March 1986 with a one month history of malaise, arthralgia and conjunctivitis followed two weeks later with cough and blood

(C) The Fellowship of Postgraduate Medicine, 1988 
streaked sputum. A chest X-ray showed patchy shadowing in the right lung base, and he was referred to hospital. On examination after admission he was febrile but there were no stigmata of infective endocarditis or vasculitis. A mitral stenotic murmur and right basal crackles were detected. His condition deteriorated rapidly with spiking fever, increasing dyspnoea and widespread crackles. A chest X-ray 4 days after the initial one showed extensive bilateral shadowing (Figure 1).

His ESR was $118 \mathrm{~mm} / \mathrm{h}$, haemoglobin $6.6 \mathrm{~g} / \mathrm{dl}$, white cell count $9.7 \times 10^{9} / 1$, urea $12.7 \mathrm{mmol} / 1$ and creatinine $193 \mu \mathrm{mol} / 1$. Pus cells, red cell casts and protein were detected in his urine. A diagnosis of pulmonary haemorrhage and glomerulonephritis (PHG) was made and he was transfused 2 units of packed red cells. Blood culture, atypical pneumonia and viral titres were negative. Examination revealed no ulceration of his nasopharynx or larynx. Renal biopsy showed crescentic glomerulonephritis with over $60 \%$ of glomeruli being involved (Figure $2 a$ ). Immunofluorescence did not show linear deposition of IgG along the glomerular basement membrane.

Because of worsening respiratory failure, he was artificially ventilated. Renal function continued to deteriorate. Haemodialysis, immunosuppressive therapy consisting of cyclophosphamide $150 \mathrm{mg}$ /day and methylprednisolone $1 \mathrm{~g} /$ day for 3 days followed by prednisolone $60 \mathrm{mg} /$ day and plasma exchange were commenced.

After intensive therapy lasting 2 weeks and further blood transfusion of 8 units, his pulmonary and renal function improved. However the white cell count dropped to $2.2 \times 10^{9} / 1$ and cyclophosphamide was stopped. X-ray showed partial clearing of the shadows and he was weaned off the ventilator. Lung function testing 7 days later showed, as percentage predicted: forced expiratory volume in one second $53 \%$, forced vital capacity $49 \%$, diffusing capacity $\left(\mathrm{D}_{\mathrm{L}} \mathrm{CO}\right) 119 \%$.

Cyclophosphamide was recommenced after 12 days. $\mathrm{He}$ was maintained on $30 \mathrm{mg}$ prednisolone daily; renal function was stable (serum creatinine $338 \mu \mathrm{mol} / \mathrm{l})$ without dialysis.

Six weeks after admission, he suddenly became dyspnoeic and coughed up large amounts of fresh blood. The haemoglobin level fell from 9 to $7.8 \mathrm{~g} / \mathrm{dl}$ and repeat lung function tests showed a rise in his $\mathrm{D}_{\mathrm{L}} \mathrm{CO}$ to $135 \%$ of predicted suggesting relapse of the disease. ${ }^{9}$ His chest X-ray deteriorated rapidly to virtual complete opacification.

Pulse therapy with methylprednisolone and cyclophosphamide were started, covered by erythromycin, cefuroxime and gentamicin. Despite artificial ventilation, gas exchange could not be maintained and the patient died 3 days later.

Permission was granted for a limited postmortem examination. This showed alveolar haemorrhage, consolidation, abscess formation in both lungs, and the presence of CMV inclusion bodies (Figure 2b) in both lungs. Sections of the kidneys showed $65 \%$ glomeruli with cellular crescents, with no immunoglobulin deposition, or vasculitis. CMV inclusion bodies were also found in the kidney, liver and pancreas.

\section{Discussion}

In the absence of anti-GBM antibody in the kidney
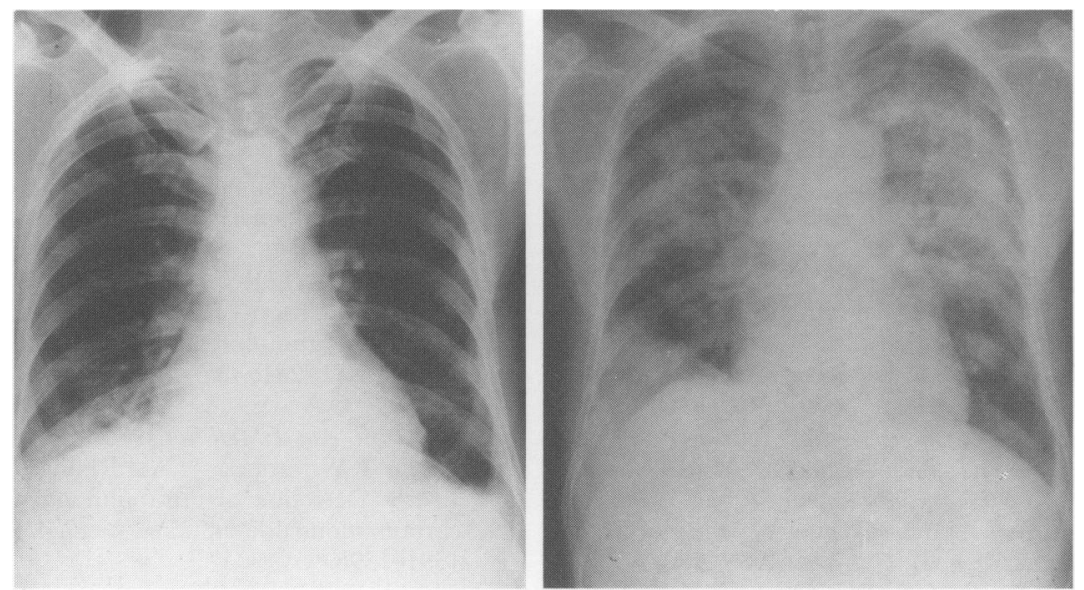

Figure 1 Chest radiographs showing right basal shadowing progressing to extensive patchy shadowing within 4 days of admission. 

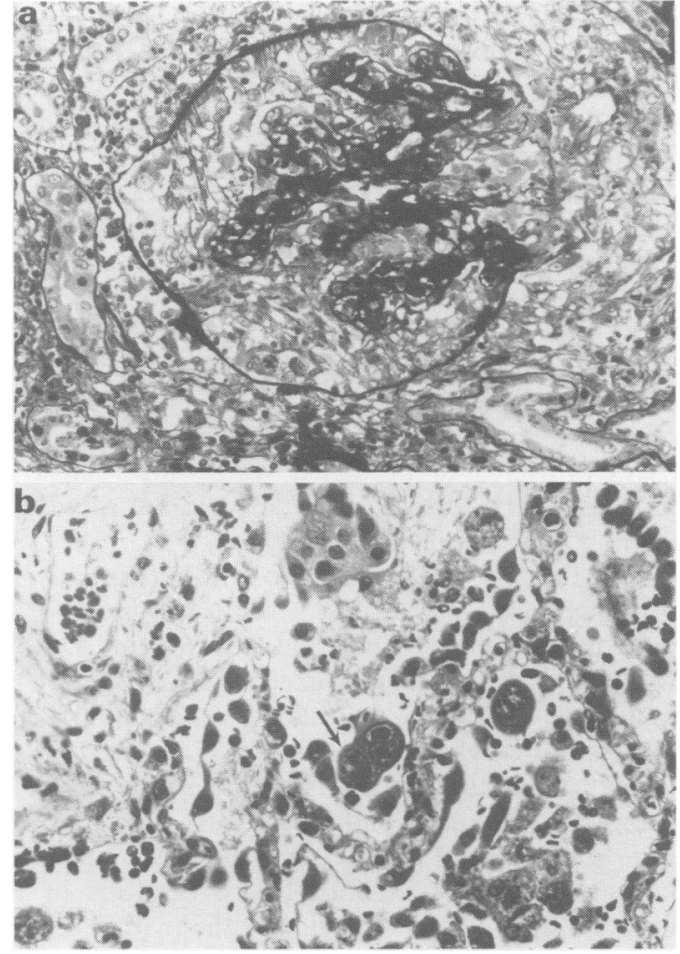

Figure 2 (a) Renal biopsy showing glomerular tufts compressed by a circumferential crescent. PASM $\times 280$. (b) Presence of CMV nuclear inclusion bodies (arrowed) and alveolar haemorrhage in both lungs at post mortem. H. \& E. $\times 100$.

and systemic vasculitic illness, this patient probably suffered from the idiopathic variety of PHG. He

\section{References}

1. Goodpasture, E.W. The significance of certain pulmonary lesions in relation to aetiology of influenza. Am J Med Sci 1919, 158: 863-870.

2. Lerner, R., Glassock, R.J. \& Dixon, F.J. The role of antiglomerular basement membrane antibody in the pathogenesis of human glomerulonephritis. $J$ Exp Med 1967, 126: 989-1004.

3. Thomas, K.M. \& Irwin, R.S. Classification of diffuse intrapulmonary haemorrhage. Chest 1975, 68: 483484.

4. Holdsworth, S., Boyce, N., Thomas, N.M. \& Atkins, R.C. The clinical spectrum of acute glomerulonephritis and lung haemorrhage (Goodpasture's syndrome). $Q J$ Med 1985, 55: 75-86.

5. Wilson, C.B. \& Smith, C.S. Goodpasture's syndrome associated with influenza $\mathrm{A}_{2}$ virus infection. Ann Intern Med 1972, 76: 91-94. improved initially on aggressive treatment. Despite stabilization of renal function, the second episode of pulmonary haemorrhage proved fatal.

Although sepsis is the main cause of death in idiopathic $\mathrm{PHG}^{4}{ }^{4}$ it was probably pulmonary haemorrhage rather than CMV infection that was the direct cause of his rapid demise. Wilson \& Smith reported a case of PHG associated with influenza $\mathrm{A}_{2}$ infection $^{5}$ and proposed that viral infection may induce antibodies reactive with the basement membrane which further perpetuated the disease. Relapse of anti-GBM disease ${ }^{6}$ and Wegener's granulomatosis ${ }^{7}$ has been associated with microbial infection, and the site of infection appears to determine the site of relapse. ${ }^{7}$ Thus, CMV infection in our patient may have a similar role in precipitating the fatal rebleed. His immunosuppressed state may have encouraged reactivation of latent virus but acquisition of infection through blood transfusion is an alternative explanation. ${ }^{8}$ The latter has been shown to occur in infants ${ }^{10}$ but can be largely eliminated by using blood from seronegative donors. ${ }^{11}$ Routine screening of donors for the virus and its antibodies is not thought to be practical but frozen deglycerolized red blood cells have been employed with success in limited trials. ${ }^{12}$

Idiopathic PHG has a relatively good long term prognosis. The majority of patients have no long term sequelae in pulmonary function and only mils to moderate renal impairment without progression at follow-up. ${ }^{4}$ In view of this, preventive measures such as screening for CMV virus before non-urgent blood transfusion may still be worthwhile in immunosuppressed patients.

6. Rees, A.J., Lockwood, C.M., Peters, D.K. Enhanced allergic tissue damage in Goodpasture's syndrome by intercurrent bacterial infection. $\mathrm{Br}$ Med J 1977, 2: 723-726.

7. Pinching, A.J., Rees, A.J., Pussell, B.A., Lockwood, C.M., Mitchison, R.S. \& Peters, D.K. Relapse in Wegener's granulomatosis: the role of infection. $\mathrm{Br}$ Med J 1981, 281: 836-838.

8. Alford, C.A. Jr. \& Charles, A. Cytomegalovirus infection. Ala J Med Sci 1985, 22: 169-189.

9. Ewan, P.W., Jones, H.A., Rhodes, C.G. \& Hughes, J.M.B. Detection of intrapulmonary hemorrhage with carbon monoxide uptake. $N$ Engl J Med 1976, 295: 1391-1396. 
10. Adler, S.P., Chandrika, T., Lawrence, L. \& Bagget, J. Cytomegalovirus infections in neonates acquired by blood transfusions. Pediatr Infect Dis 1983, 2: 114 118.

11. Yeaner, A.S., Grumet, F.C., Hafleigh, E.B., Arvin, A.M., Bradley, J.S. \& Prober, C.G. Prevention of transfusion acquired cytomegalovirus infections in newborn infants. $J$ Pediatr 1981, 98: 281-287.
12. Brady, M.T., Milam, J.P., Anderson, D.C. et al. Use of deglycerolized red blood cells to prevent post transfusion cytomegalovirus infection in neonates. $J$ Infect Dis 1985, 150: 334-338. 